\title{
TETRAHEURÍSTICA SISTÉMICA (THS) PARA EL TSP
}

SYSTEMIC TETRAHEURISTIC FOR THE TSP

\author{
Jorge Iván Pérez Rave ${ }^{1} \quad$ Gloria Patricia Jaramillo Álvarez ${ }^{2} \quad$ Carlos Mario Parra Mesa $^{3}$ \\ Luis Fernando Moreno Velásquez ${ }^{4}$
}

Recibido 23 de junio de 2009, aceptado 13 de julio de 2010

Received: June 23, 2009 Accepted: July 13, 2010

\begin{abstract}
RESUMEN
Este artículo presenta un novedoso método, basado en elementos del pensamiento sistémico, para solucionar instancias del problema del vendedor viajero (TSP), el cual es comparado en términos de eficacia y eficiencia con "nearest neighbour", "cheapest insertion", "two-way exchange improvement" y "branch and bound". El primer apartado introduce la optimización combinatoria, el segundo ofrece un marco de referencia, el tercero presenta la metodología empleada, el cuarto apartado presenta el desarrollo de la tetraheurística sistémica, seguido del análisis de varianza y de rangos de Duncan para los factores: método y cantidad de ciudades; este apartado finaliza con el análisis del comportamiento de la proporción de "fracasos" del algoritmo propuesto a medida que aumenta la complejidad del TSP. Como resultado, se obtiene un método para resolver instancias del TSP, conformado por tres heurísticas misionales: 1. "vecino más cercano", 2. "sacrificio cortoplacista" y 3. "traslado LIFO", y una de apoyo llamada "búsqueda derecha 4P4". El diseño de la heurística denominada "sacrificio cortoplacista" es inspirado en el análisis sistémico del "vecino más cercano", al cual se le identifica el arquetipo de "soluciones rápidas que fallan", con aplicación a decisiones cotidianas. La tetraheurística sistémica se destaca, respecto a las demás, en solución arrojada y en tiempo computacional consumido, especialmente cuando incrementa la complejidad del TSP.
\end{abstract}

Palabras clave: Optimización combinatoria, tetraheurística, pensamiento sistémico, problema del vendedor viajero.

\begin{abstract}
This paper presents a novel method to solve instances of the TSP. This method is comparable in effectiveness and efficiency with "nearest neighbour", "cheapest insertion", "two-way exchange improvement" and "branch and bound". The first section provides a literature review of the combinatorial optimization, the second provides a reference frame, the third the methodology used and the fourth contains, inter alia, system thinking, AxB factorial design and management tool CAP-DO. The fourth section presents the design "new" method called systemic tetraheuristic, followed by ANOVA and Duncan ranges for each factor: method and number of cities; this section concludes with an analysis of the proportion of "failures" of the proposed algorithm with increasing complexity of the TSP. As a result of this study a method is presented for solving the TSP. This method consists of: 1. "nearest neighbour", 2. "short-term sacrifice" 3. "LIFO transfer" and a support heuristic "right search 4P4 ". For the design of the "sacrifice short-term" procedure, it was necessary to analyze the "nearest neighbour" heuristic from a systems perspective. This heuristic is related to the "quick solutions that fail" archetype, which can be applied to day-to-day. The proposed method has proved to be more efficient than others, in what concerns quality of the solution and the computation time, especially when the complexity of the TSP increases.
\end{abstract}

Keywords: Combinatorial optimization, tetraheuristics, systems thinking, travelling salesman problem.

1 Departamento de Ingeniería Industrial. Universidad de Antioquia. Grupo de Investigación Gestión de la Calidad. Calle 67 No 53·108, B1 21, of. 404. Medellín, Colombia. E-mail: ejipr056@udea.edu.co

2 Escuela de Sistemas. Laboratorio de Análisis y Modelamiento Territorial LAMAT. Instituto de Sistemas y Ciencias de la Decisión. Facultad de Minas. Universidad Nacional de Colombia. Medellín, Colombia. E-mail: gpjaramia@ gmail.com

3 Departamento de Ingeniería Industrial. Universidad de Antioquia. Grupo de Investigación Gestión de la Calidad. Medellín, Colombia. E-mail: cmparra@udea.edu.co

4 Escuela de Sistemas. Facultad de Minas. Universidad Nacional de Colombia. Medellín, Colombia. E-mail: 1fmoreno@unalmed.edu.co 


\section{INTRODUCCIÓN}

La investigación de operaciones conocida como ciencia de la toma de decisiones tiene décadas de existencia y, si bien tiene su origen en aplicaciones militares [1], cobra cada vez mayor rigor en las decisiones empresariales, tanto en el ámbito de producción como en servicios [2-4].

Dentro de las necesidades empresariales a las que puede aportarse con el uso de esta rama se encuentran los problemas de optimización combinatoria, caracterizados porque las variables de decisión son de tipo discreto y sus diferentes soluciones se forman a raíz de la ordenación de números enteros. A pesar de la relevancia de esta disciplina, su aplicación a problemas reales no es tan simple como seguramente se desea, debido a la cantidad de variables y limitantes que rodean una decisión empresarial en términos combinatorios.

Bajo este contexto retador, quizá uno de los problemas más famosos y complejos de la optimización combinatoria es el "agente viajero" o TSP (traveling salesman problem). Este problema es considerado en el ámbito científico y empresarial como una referencia "obligada" para validar cualquier método de resolución de problemas de optimización combinatoria tipo NP-hard [5-6].

En la actualidad, son innumerables los procedimientos desarrollados para aportar a la optimización combinatoria, clasificados en diversas categorías que posteriormente se detallarán, pero en este artículo se tiene particular interés en cuatro de ellas, incluidas en el clásico y empleado software WinQSB [7]: "nearest neighbour", "cheapest insertion", two-way exchange improvement" y "branch and bound method", con las cuales se pretende comparar en términos de eficacia y eficiencia un método desarrollado por los autores, basado en el pensamiento sistémico, para aportar soluciones al famoso "agente viajero".

De resultar benéfico en términos de simplicidad, novedad, calidad de la respuesta encontrada y tiempo computacional, el método desarrollado ofrecería elementos de apoyo tanto para la comunidad científica como empresarial, para continuar en la búsqueda de soluciones más efectivas a los muchos problemas reales, así como teóricos.

\section{MARCO DE REFERENCIA}

Desde 1958 Simon y Newell avecinaron el auge de los algoritmos heurísticos en la solución de problemas complejos, propios del campo de la investigación de operaciones [8] y, efectivamente hoy día, son amplias sus aplicaciones, que inician en campos militares y se trasladan hacia la industria y los servicios [9-10].
El término heurística deriva del griego Heuriskein, que significa descubrir o encontrar y en el campo de la optimización engloba una clase de procedimientos de resolución de problemas. Estos procedimientos pueden entenderse como una serie de herramientas que por medio de procedimientos algorítmicos sistemáticos, uso de la creatividad, el sentido común y la imaginación, pero también con elementos propios de las ciencias básicas, permiten encontrar "buenas" soluciones a problemas complejos y en un tiempo computacional razonable desde el punto de vista práctico.

Una de las subdisciplinas de la investigación de operaciones, en la que este tipo de procedimientos tiene amplio uso, es la optimización combinatoria, donde la mayoría de sus problemas se consideran "difíciles" de resolver por medio de métodos exactos [11-12], pues poseen variables de tipo discreto, el espacio de soluciones está conformado por un subconjunto finito de números naturales o por la secuenciación de los mismos y comúnmente, desde el punto de vista práctico, la exploración de todas las posibilidades amerita exagerado tiempo computacional, que hace inviable obtener una solución óptima.

Dentro de los problemas más estudiados en esta categoría de complejidad se encuentra el "agente viajero" o traveling salesman problem (TSP) y, para ilustrarlo, supóngase que un vendedor se encuentra en la ciudad $1 \mathrm{y}$, partiendo de ésta, debe realizar un tour abordando cuatro ciudades más para ofrecer sus productos, entendiendo como tour el evento de que visite cada ciudad una sola vez, y al final, regrese a la de origen. En la Figura 1 se ilustran dos posibles tours para el citado ejemplo.
TOUR A
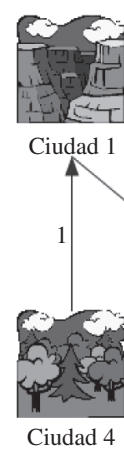

Ciudad 4
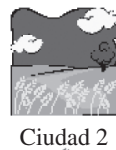

(1)

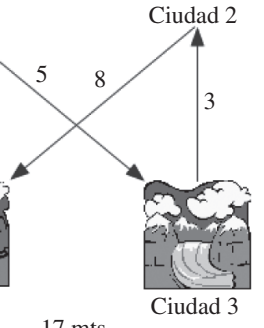

$17 \mathrm{mts}$
Figura 1. Ejemplo de dos tours para un TSP de cuatro ciudades.

Fuente: Elaboración propia.

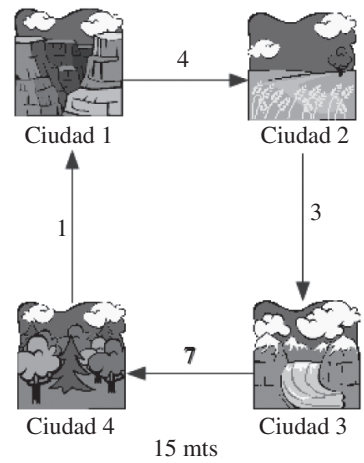

$15 \mathrm{mts}$ 
El tour A sigue la ruta: 1, 3, 2, 4 y regresa a 1, lo que conlleva recorrer una distancia total de 17; o también podría generarse un segundo tour (B) recorriendo: 1, 2, 3, 4 y desde allí regresa a 1 , haciendo esta vez 15 . El objetivo final es recorrer el tour completo en la menor distancia posible.

Para este sencillo ejemplo, asumiendo simetría, deben explorarse sólo 12 alternativas, que en un computador actual tardaría simples fracciones de segundo; no obstante, cuando el problema se asemeja a la realidad, por ejemplo, teniendo 50 ciudades con distancias asimétricas, ameritaría explorar 6,08282 $\times 10^{62}$ alternativas, que en tiempo de las computadoras actuales representaría algo tan abismal que se sale de la viabilidad práctica. De manera general esta función de tiempo computacional crece de forma factorial a medida que aumenta el número de ciudades [13].

Este problema, publicado por Flood en el año 1956 [14], clásico de la optimización combinatoria, es simple de formular en términos matemáticos [15-16], pero su resolución, como se nota, es sumamente compleja. Desde su lanzamiento, hasta la fecha, existe abundante literatura sobre algoritmos probados en el TSP entre ellos [17-19].

La aplicación de heurísticas al TSP aborda variedad de campos de acción, entre los que cabe mencionar: programación de tareas en entornos manufactureros [20], fabricación de circuitos integrados, asignación de rutas para un vehículo, recogida (robotizada) de materiales almacenados, instalaciones de partes en ordenadores, así como subproblemas de otros casos más extensos [21] como el problema de ruteo de vehículos; igualmente: programación de servicios de llamadas, optimización de las trayectorias de la herramienta en un equipo manufacturero y un trazador de gráficos para dibujar una figura dada [22].

La diversidad de algoritmos heurísticos para aportar soluciones al TSP comúnmente puede agruparse en constructivos, de descomposición, de reducción, de manipulación y de búsqueda local [23-24]. Se hará hincapié conceptual en los métodos constructivos y los de búsqueda local, pues son los asociados a los algoritmos con que se pretende comparar el método desarrollado por los autores:

- Constructivos: Consisten en ir agregando elementos individuales hasta completar una solución viable. Generalmente se basan en asignar el "mejor" elemento en cada iteración. Entre los clásicos aplicados al TSP se encuentra la heurística del "Vecino más cercano".

- De mejoramiento: A diferencia de los otros tipos, en estos procedimientos se parte de una solución de inicio, comúnmente obtenida a través de otra heurística, y a partir de ella se generan otras, de modo que con cada modificación se arroja un resultado mejor que el anteriormente obtenido, se actualiza la respuesta del algoritmo, repitiendo el paso hasta un determinado criterio de parada.

Para resolver instancias "NP-hard" del TSP, aparte de los algoritmos heurísticos, los cuales forman parte de la "clase aproximada", se cuenta también con métodos basados en la enumeración, pero desde luego no completamente exhaustiva, pues, como se mencionó, carecería de viabilidad práctica. El método de enumeración más empleado para resolver instancias del TSP es "branch and bound", donde la "ramificación" corresponde a la enumeración y el "acotamiento" al descarte de soluciones posibles en comparación con una cota superior o inferior [25], lo cual es crítico para evitar la enumeración exhaustiva.

En el presente artículo, en cuestión de métodos aproximados, se hará énfasis en los constructivos: "nearest neighbour" [26] y "cheapest insertion" [27] y, en heurísticas de mejoramiento: "two-way exchange improvement" [28]; así como, en el caso de métodos enumerativos "branch and bound", todos ellos incluidos en el WinQSB en el módulo de resolución al TSP, y contra estos se comparará la eficacia y eficiencia del método propuesto.

\section{Nearest neighbour heuristic (vecino más cercano)}

Este algoritmo hace parte de los métodos constructivos y se basa en una elección cortoplacista de lo más cercano desde el lugar o punto donde se encuentra el viajero. Al principio del viaje las distancias se tornan cortas, pero a medida que el viajero avanza debe elegir entre ciudades antes no seleccionadas que pueden acrecentar la distancia total recorrida, sobre todo lo generado al regreso.

\section{Cheapest insertion (inserción más barata)}

Bajo este procedimiento se inicia en cualquier ciudad y se determina su vecina más próxima, por ejemplo, considere una instancia del TSP con cuatro ciudades cuya matriz de distancias simétricas se muestra en la Tabla 1.

Tabla 1. Instancia del TSP con cuatro ciudades.

\begin{tabular}{|c|c|c|c|c|}
\cline { 2 - 5 } \multicolumn{1}{c|}{} & $\mathbf{1}$ & $\mathbf{2}$ & $\mathbf{3}$ & $\mathbf{4}$ \\
\hline $\mathbf{1}$ & ---- & 9 & 2 & 3 \\
\hline $\mathbf{2}$ & 9 & ----- & 1 & 4 \\
\hline $\mathbf{3}$ & 2 & 1 & ---- & 8 \\
\hline $\mathbf{4}$ & 3 & 4 & 8 & ---- \\
\hline
\end{tabular}


Seleccionando a priori la ciudad 1, la más cercana es la ciudad 3; así entonces, se crea un primer subtour. Posterior a ello, se analiza cuál de las dos rutas $(1,3)$, $(3,1)$ es conveniente reemplazar, con miras a disminuir la distancia al adicionar otra ciudad al tour actual. En la Tabla 2 se muestran las distancias para las rutas a evaluar con la inclusión de cada una de las demás ciudades.

Tabla 2. Ejemplo de un paso del método de inserción más barata.

\begin{tabular}{|c|c|c|}
\hline Rutas a evaluar & Rutas a adicionar & Distancia \\
\hline$(1,3)$ & $(1,2)(2,3)$ & $9+1-2=8$ \\
\hline$(1,3)$ & $(1,4)(4,3)$ & $3+8-2=9$ \\
\hline$(3,1)$ & $(3,2)(2,1)$ & $1+9-2=8^{*}$ \\
\hline$(3,1)$ & $(3,4)(4,1)$ & $8+3-2=9$ \\
\hline
\end{tabular}

A partir de este ejercicio se nota en la Tabla 2 que, para aumentar el subtour con una ciudad más, conviene descartar la ruta $(3,1)$ o $(1,3)$ e incluir en medio de ésta la ciudad 2, ya que produce menor distancia que si se incluye una de las demás. Eligiendo de manera arbitraria la ruta $(3,1)$ se construye el subtour $(3,2,1)$. El procedimiento continúa, pero esta vez se evalúa la pertinencia de cada una de las rutas del subtour actual $(3,2),(2,1)$ y $(1,3)$ al adicionar la ciudad 4 , y de nuevo se selecciona el tour de menor distancia. El resto del procedimiento se deja como ejercicio para el lector, anunciando que la respuesta arrojada bajo esta heurística es $(3,2,4,1,3)$ con una distancia de 10 .

\section{Two-way exchange improvement heuristic (2- intercambio)}

Este procedimiento forma parte de los métodos de mejoramiento. En él se parte de un tour cualquiera, que bien puede ser escogido a través de otro algoritmo, y a partir de allí genera iteraciones en busca de la mejora de la solución de inicio. Para ello se apoya en la premisa de que si en un determinado tour dos rutas se cruzan entre sí, es posible reconectarlas por una unión entre las ciudades implicadas que evita el cruce entre ellas, existiendo para cada caso una sola manera de hacerlo. Esta operación conlleva a disminuir la distancia del nuevo tour generado con relación al anterior. En la Figura 2 se observa un tour inicial (A) donde se cruzan dos rutas y a su lado el tour "B" generado a partir de un movimiento de 2-intercambio.

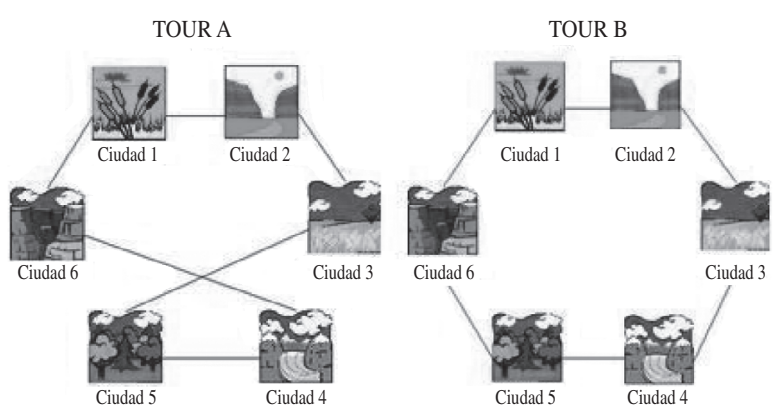

Figura 2. Ejemplo de 2-intercambio en una instancia de TSP con seis ciudades.

Fuente: Elaboración propia.

El hecho de que este algoritmo explore todos los vértices para realizar el 2-intercambio, de modo que en cada paso se seleccione el cambio que más mejore la solución actual, puede afectar positivamente la eficacia del algoritmo, pero a su vez reducir su eficiencia. Para mitigar su impacto en la eficiencia, comúnmente, en lugar de explorar todos los vértices se considera sólo una lista de candidatos. Para mayor información sobre este procedimiento se recomienda leer [29-30].

\section{Branch and bound (ramificación y acotamiento)}

El TSP puede formularse como un típico problema de asignación, pero, además, es preciso agregar restricciones para impedir que la solución final conste de subtours. Para profundizar en estas restricciones remítase a [31-32]. Por ejemplo, para una instancia de cinco ciudades para el TSP, sin incluir estas restricciones puede llegarse quizá a una solución inviable [33] como se muestra en la Figura 3.

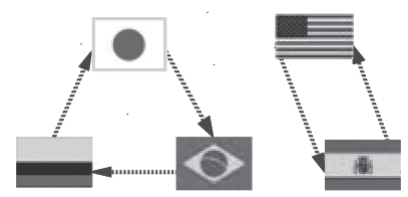

Figura 3. Solución inviable TSP debido a subtours. Fuente: Elaboración propia.

En forma general, lo que realiza el algoritmo de ramificación y acotamiento para el TSP es iniciar con la solución del problema de asignación respectivo y si ésta corresponde a un tour se detiene, en caso contrario, introduce restricciones que eliminan estos subtours [34].

Hasta ahora se ha hecho énfasis en sustento literario sobre inicios, definiciones, tipos, entre otros, pero queda un interrogante quizá, y es precisamente ¿cómo determinar si la solución arrojada por uno u otro método puede considerarse satisfactoria? 
Las respuestas a este interrogante dependen en gran parte de las características específicas de cada problema, entre ellas: las unidades de medida, pues no es lo mismo hablar de una distancia que puede representarse a nivel práctico en simples metros, a una que amerita millas; así como hay notoria diferencia entre inversiones de miles de pesos y las de millones de dólares. Dependiendo de ello, el decisor puede interesarse en esperar hasta días con tal de obtener una solución casi exhaustiva; igualmente, otro puede ser el caso y ser interesante también la respuesta casi de manera inmediata.

En términos generales, en un método heurístico subyacen dos variables fundamentales: la eficacia y la eficiencia. La primera puede interpretarse como el logro del objetivo; es decir, que encuentre, si no es la solución óptima, una muy cercana a ello. La segunda hace referencia a que arroje una respuesta racionalizando los recursos, que en este caso equivalen al tiempo de cómputo. Con base en la eficacia y eficiencia, comúnmente se llevan a cabo comparaciones entre procedimientos aproximados y hasta enumerativos empleados en la optimización combinatoria. Estas, a su vez, pueden realizarse mediante la comparación con: la respuesta óptima (cuando se conoce), una cota, un método exacto, o bien con otro algoritmo [35]. Este último constituye el procedimiento a emplear para comparar el método desarrollado, que tiene por nombre tetraheurística sistémica (THS).

\section{METODOLOGÍA}

\section{Fase 1. Diseño y desarrollo del método}

Con base en las características del TSP y en la literatura, se procedió a chequear, para instancias menores del TSP (entre 4 y 10 ciudades), las soluciones arrojadas por los cuatro procedimientos de comparación: "nearest neighbour", "cheapest insertion", "two-way exchange improvement" y "branch and bound".

Luego se continuó con el ciclo gerencial CAP-DO (chequear, analizar, planear y hacer) [36], a fin de generar estrategias, siempre procurando una visión sistémica de los casos, en busca de cercanías a la "mejor" respuesta arrojada por las heurísticas referentes. A medida que esto se logra, se amplió la complejidad de las instancias TSP. De este modo, con elementos de: sentido común, imaginación, creatividad, programación en Macros-Visual Basic, ciencias básicas y pensamiento sistémico, se hizo girar continuamente el ciclo CAP-DO, construyendo diversas versiones del método a desarrollar, deteniendo el giro del ciclo al obtener resultados satisfactorios para el equipo investigador, en términos de eficacia y eficiencia de la heurística.
Fase 2. Comparación con los procedimientos en distancia/costo y tiempo computacional

En esta fase se buscó determinar si existe diferencia, en términos de distancia/costo y de tiempo de cómputo, entre el método propuesto por los autores y los referentes; de ser así, conocer con cuál(es) de ellos se presentan diferencias. Asimismo, es de interés conocer si existe interacción entre el método y la complejidad del problema en términos del número de ciudades. Para cumplir con los objetivos de esta fase se recurrió al diseño factorial $\mathrm{AxB}$ con dos observaciones de cada experimento [37], donde "A" corresponde al método y "B" a la cantidad de ciudades. Los niveles de los factores "A" y de "B" se presentan en la Tabla 3.

Tabla 3. Factores y sus niveles en la fase 1.

\begin{tabular}{|c|c|c|c|}
\hline Factor & Niveles & Descripción & Notación \\
\hline \multirow{4}{*}{ (A) Método } & \multirow{2}{*}{5} & $\begin{array}{c}\text { Tetraheurística } \\
\text { sistémica }\end{array}$ & THS \\
\cline { 3 - 4 } & & Nearest neighbour & Nearest N \\
\cline { 3 - 4 } & & Cheapest insertion & Cheapest I \\
\cline { 3 - 4 } & $\begin{array}{c}\text { Two-way exchange } \\
\text { improvement }\end{array}$ & Two-way EI \\
\cline { 3 - 4 } & Branch and bound & Branch B \\
\hline (B) $\mathrm{N}^{\circ}$ de ciudades & 23 & $6,12,24, \ldots, 138$ & $6,12,24, \ldots, 138$ \\
\hline
\end{tabular}

Para este caso se tienen dos diseños factoriales, cuya diferencia entre ambos es que uno posee como variable respuesta el tiempo de cómputo en segundos y el otro la distancia/costo arrojada por cada algoritmo. Cabe anotar que en el software WinQSB no fue posible obtener respuestas para instancias del TSP superiores a 143 ciudades.

Para la experimentación, de cada ciudad se generaron dos instancias diferentes del TSP, cuyos datos de costos/ distancias provienen de una distribución uniforme entre 0 y 100 construida a partir de la función de números pseudoaleatorios del Excel. De este modo, cada método se probó dos veces en cada ciudad, analizando tanto la distancia/costo arrojado por cada uno y el tiempo de cómputo consumido en un computador Intel(R) Pentium 4 de CPU 3.06GHz, $1 \mathrm{~Gb}$ de RAM. Esta primera etapa metodológica consumió: $23 \times 5 \times 2=230$ experimentaciones. Cada una de las observaciones del experimento factorial puede representarse mediante el siguiente modelo:

$$
y_{i j k}=\mu+\tau_{i}+\beta_{j}+(\tau \beta)_{i j}+\varepsilon_{i j k}
$$

Donde i $=1,2, \ldots, 5$ (Método); $\mathrm{j}=6,12,24, \ldots, 138$ (cantidad de ciudades); $\mathrm{y} \mathrm{k}=1,2$ (observaciones).

De este modo, $y_{i j k}$ representa la respuesta arrojada (distancia/costo y similar cuando se trabaja con tiempo 
computacional) por el i-ésimo método cuando es probado con una instancia del TSP de j ciudades en la k-ésima observación del experimento. $\mu$ hace alusión al efecto medio global, $\tau_{i}$ es el efecto que ejerce el nivel i-ésimo del factor método y $\beta_{j}$ equivale al efecto del nivel j-ésimo del factor cantidad de ciudades. Asimismo, $(\tau \beta)_{i j}$ corresponde al efecto de la interacción entre los factores A y B y, finalmente, $\varepsilon_{i j k}$ es el error aleatorio.

Generados los experimentos, con el Statgraphics 5.1 se realizó la prueba de análisis de varianza [38] para determinar si existe diferencia entre los métodos sometidos a ensayo, tanto en tiempos de cómputo como en distancias/costos. Igualmente, esta prueba se aplica para analizar el efecto del factor tamaño de las ciudades y para la interacción AxB. De comprobarse diferencias, se procederá a aplicar Rangos múltiples de Duncan [39], de modo que se identifique entre qué niveles de los factores específicos existen diferencias.

Tanto las pruebas estadísticas aplicadas como los análisis, se realizaron, para cada diseño AxB, de manera individual; es decir, primero se abordó para las distancias/costos y luego para el tiempo de cómputo.

\section{Fase 3. Análisis de la proporción de "fracasos"} Tetraheurística Sistémica (THS)

En esta fase se pretende estimar p: "proporción de fracasos del método desarrollado", entendiéndose el término "fracaso" como el evento de que al menos uno de los procedimientos referentes arroje menor distancia/costo que THS. Igualmente, se busca analizar el comportamiento de dicha proporción a medida que las instancias del TSP son más complejas (aumentando las ciudades). El cálculo de esta proporción se basa en lo siguiente: sea $X_{j}$ la variable aleatoria que representa el evento de "fracaso" cuando THS es probada en el j-ésimo nivel del factor número de ciudades. Si esto ocurre, $X_{j}$ tomará el valor de 1 , en caso contrario su valor será 0 . De este modo, la función de densidad de probabilidad $f\left(x_{j}\right)$ corresponde a la distribución Bernoulli, la cual viene dada por:

$$
\begin{gathered}
P\left(X_{j}=0\right)=\left(1-p_{j}\right) \\
P\left(X_{j}=1\right)=p_{j}
\end{gathered}
$$

Con $X_{j}: 0,1$, para j: niveles del factor ciudad.

Para cada nivel del factor ciudades, el parámetro $p_{j}$ se estima así:

$$
p_{j}=\frac{\sum_{j=1}^{N_{j}} X_{j}}{N_{j}}
$$

Donde $N_{j}$ es el número total de pruebas realizadas hasta el j-ésimo nivel del factor número de ciudades.

\section{RESULTADOS Y ANÁLISIS}

\section{Fase 1. Diseño y desarrollo del método}

En la Figura 4 se presenta a nivel macro el modelo mental del método desarrollado.

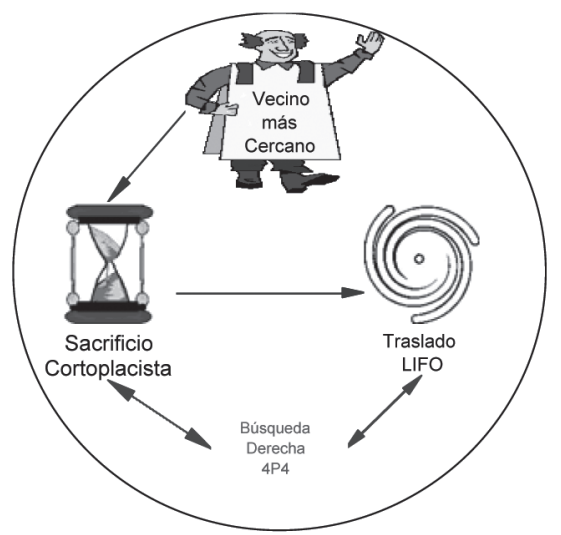

Figura 4. Esquema de THS.

Fuente: Elaboración propia.

El THS consta de tres etapas misionales y una de apoyo. La primera se refiere al típico vecino más cercano, en tanto que las demás son desarrollos del equipo investigador, denominados: sacrificio cortoplacista, traslado LIFO, ambos con una fase de apoyo por nombre "búsqueda derecha 4P4". A través de un ejemplo, se socializará paso a paso cada una de las etapas que integran la tetraheurística. Para ello, considere la matriz de distancias que se muestra en la Tabla 4, la cual representa una instancia del TSP para seis ciudades.

Tabla 4. Instancia del TSP para seis ciudades con distancias simétricas.

\begin{tabular}{|c|c|c|c|c|c|c|}
\cline { 2 - 7 } \multicolumn{1}{c|}{} & $\mathbf{1}$ & $\mathbf{2}$ & $\mathbf{3}$ & $\mathbf{4}$ & $\mathbf{5}$ & $\mathbf{6}$ \\
\hline $\mathbf{1}$ & 0 & 52 & 32 & 94 & 15 & 68 \\
\hline $\mathbf{2}$ & 52 & 0 & 86 & 67 & 73 & 78 \\
\hline $\mathbf{3}$ & 32 & 86 & 0 & 77 & 83 & 71 \\
\hline $\mathbf{4}$ & 94 & 67 & 77 & 0 & 57 & 60 \\
\hline $\mathbf{5}$ & 15 & 73 & 83 & 57 & 0 & 83 \\
\hline $\mathbf{6}$ & 68 & 78 & 71 & 60 & 83 & 0 \\
\hline
\end{tabular}

- Vecino más cercano (nearest neighbour) Al implementar esta heurística de construcción se obtiene una solución de inicio consistente en el tour (1, 5, 4, 6, 3, 2, 1), arrojando una distancia total de 341 . 


\section{- Sacrificio cortoplacista}

Esta busca mejorar la solución del "vecino más cercano", basándose en lo siguiente:

En la Figura 5 puede verse que en las primeras asignaciones bajo el vecino más cercano el crecimiento acumulado de la distancia/costo es relativamente mínimo, lo cual resulta satisfactorio para el decisor, y que, además, se asemeja a situaciones donde se piensa sólo en estar bien en el corto plazo; no obstante, esas decisiones "rápidas", enfocadas en el hoy, comienzan a generar un efecto dominó, que, con un retardo temporal, repercuten negativamente en lo que precisamente se desea evitar, es decir, incrementan a futuro de una manera acelerada la distancia/costo total que debe recorrer el viajero.

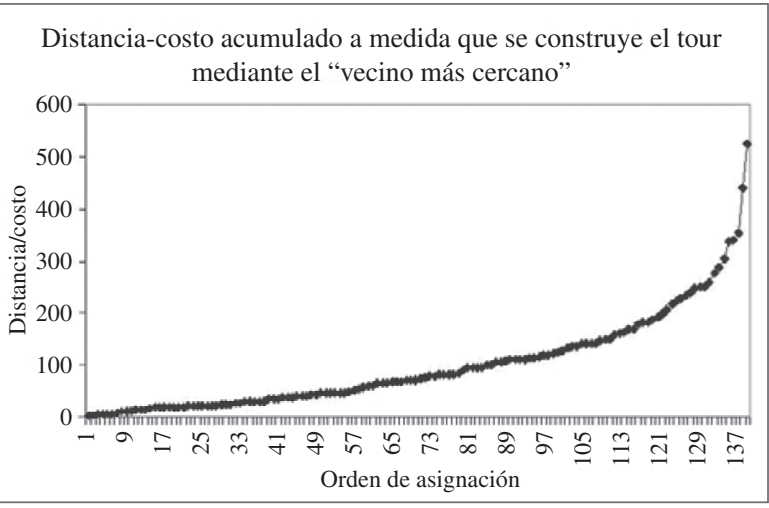

Figura 5. Distancia/costo acumulado de tour para TSP de 140 ciudades con "vecino más cercano". Fuente: Elaboración propia.

Bajo esta mirada, muy acorde a como, comúnmente, funcionan las decisiones en entornos sociales, empresariales y académicos, particularmente en la cultura occidental, se nota la importancia de adoptar un pensamiento sistémico en la cotidianidad, pues hasta en un aparentemente simple "programa de viajes" cobran relevancia las leyes del pensamiento sistémico. Así entonces, adoptando una mirada sistémica, este fenómeno se asemeja al arquetipo de "soluciones rápidas que fallan", plasmado en la Figura 6.

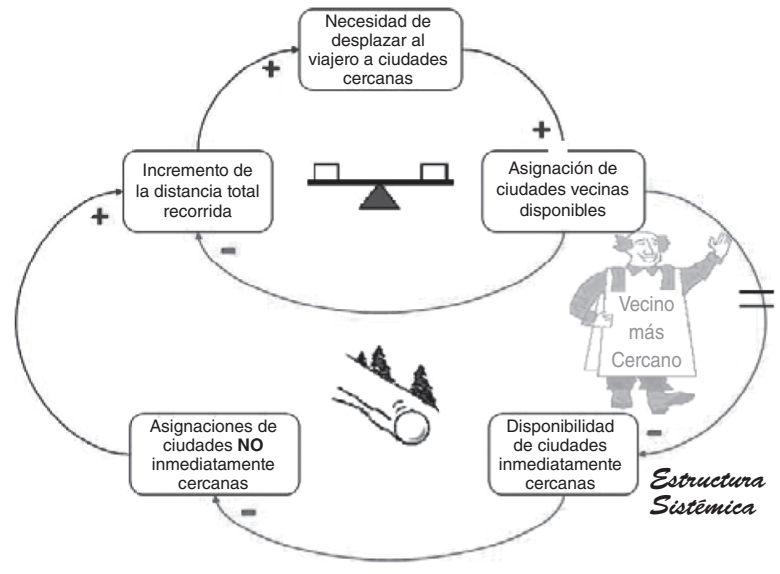

Figura 6. Representación sistémica del "vecino más cercano".

Fuente: Elaboración propia.

A partir de la Figura 6, se notan dos ciclos, uno con realimentación estable y otro con efecto de refuerzo. Una manera de interpretar esta estructura es la siguiente:

A medida que incrementa la distancia total consumida por el viajero (acumulada), a corto plazo se siente mayor la necesidad de que éste se desplace a ciudades cercanas al punto donde se encuentra, lo que motiva a emplear la estrategia del "vecino más cercano", materializándose con la asignación de ciudades vecinas que arrojan de inmediato un leve incremento en la distancia total recorrida. Con esta estrategia, en el corto plazo, a través de la solución rápida del "vecino más cercano" se pensaría que todo está controlado.

No obstante, a medida que este criterio prevalece, en el largo plazo, lo cual se ilustra con efecto retardante (II) del ciclo más externo de la Figura 6, se disminuye la disponibilidad de ciudades inmediatamente cercanas a un punto determinado donde se encuentre el viajero, pues éstas ya fueron seleccionadas en rutas anteriores. Esta consecuencia evidenciada en el largo o mediano plazo produce un efecto no deseado en el sistema, ya que "obliga" al viajero a que, en las siguientes visitas, se dirija a ciudades no inmediatamente cercanas a cada punto donde se encuentra. Al final, como consecuencia de ello, se genera con más fuerza un crecimiento en la distancia/costo total asociado al tour completo que realiza el viajero (véanse también las últimas asignaciones de la Figura 5).

Este análisis deja muchas enseñanzas a la vida diaria, donde quizá una de ellas sea "hacer sacrificios en el corto plazo esperando tener un mañana mejor", y es precisamente en esta premisa en la que se basa una de las heurísticas 
propuestas que integran el método completo desarrollado por los autores.

De ser válida la premisa de "sacrificio cortoplacista", una posible solución para que al agente viajero le vaya bien "hoy" y minimice el impacto negativo de sus decisiones en el "mañana" es renunciando a uno o más de los beneficios a los que puede acceder en el corto plazo; en otras palabras, deberá dejar de trasladarse a una ciudad inmediatamente cercana con la esperanza de que en el mañana pueda emplear esta ciudad para acortar distancias o disminuir costos.

Aparte de lo anterior, es preciso aprovechar las ventajas que en el corto plazo ofrece el procedimiento del "vecino más cercano" y generar modificaciones mínimas, una por una, a fin de identificar puntos de apalancamiento, de modo que con un pequeño cambio se genere un significativo impacto favorable en el sistema en conjunto. Con miras a ello, podría pensarse en hacer un "sacrificio de corto plazo", renunciando en cada ciudad a su vecino más cercano para recurrir a la otra mejor alternativa que le sigue.

Para una mejor ilustración de este procedimiento retómese la solución inicial del ejemplo que se viene desarrollando, la cual resulta de aplicar el "vecino más cercano". Apoyándose en la Figura 10, se nota que, partiendo del tour de inicio, se procede a analizar la primera ciudad a visitar desde el punto de origen (ciudad 1), que en este ejemplo corresponde a la ciudad $5(1,5)$. Siendo esta la mejor opción a corto plazo, se hace el "sacrificio" de renunciar a ese desplazamiento para avanzar hacia la segunda alternativa mejor a corto plazo, con la esperanza de que en el mediano-largo plazo el sistema en conjunto mejore.

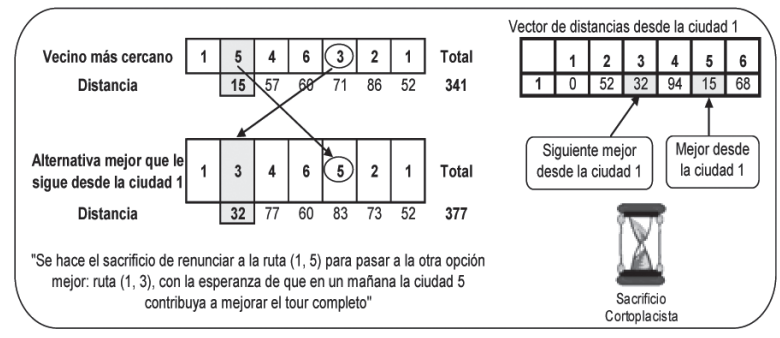

Figura 7. Ejemplo Primer intercambio "sacrificio cortoplacista".

Fuente: Elaboración propia.
El hecho de pasar hacia la inmediatamente "mejor" alternativa se basa en la intención de procurar conservar las ventajas de corto plazo arrojadas por la solución de inicio y de identificar tan sólo un pequeño cambio que genere un efecto de palanca en el sistema; es decir, permita reducir en lo posible la distancia/costo arrojada por el tour completo. Bajo esta mirada, como se nota en la Figura 7 (vector de distancias), la siguiente alternativa mejor a la que puede avanzar desde la ciudad 1 es la ciudad 3, arrojando dicha ruta un costo/distancia de corto plazo de 32, en lugar de los 15 anteriores.

A raíz de este "sacrificio cortoplacista" ya se cuenta con una segunda solución viable al TSP, que en este caso aparentemente genera una mayor distancia/costo que la proporcionada por el "vecino más cercano". Cabe anotar que la solución obtenida a través del descrito intercambio no genera pérdida de memoria de la arrojada por el "vecino más cercano"; este tema posteriormente se detallará.

Luego de este cambio, obtenido a través de "sacrificio cortoplacista", es preciso dar tiempo a que el sistema genere sus modificaciones adaptativas, similar a como ocurre en cualquier fenómeno real. Por ejemplo, cuando en una empresa se implementa una estrategia de mejora encontrará personas que la siguen y otras, con virus de la actitud, que generan turbulencias. Luego de un tiempo, se producen modificaciones evolutivas del sistema que bien pueden materializarse en el éxito o en el fracaso de la misma. Este comportamiento evolutivo trata de representarse en el "sacrificio cortoplacista" por medio de "búsqueda derecha 4P4", la cual genera modificaciones al sistema de izquierda a derecha a través de permutaciones de 4, es decir, generando 24 alternativas al intercambiar subtours conformados por 4 ciudades adyacentes a la solución de partida, permaneciendo lo demás constante.

Es de anotar que se probó con permutaciones de dos y de tres, pero con cuatro se obtuvieron resultados más satisfactorios para el equipo investigador. Con cinco no se consideró permitente probar debido a la afectación que podría sufrir la eficiencia del algoritmo, al pasar de 24 posibilidades a 120. En la Tabla 5 se muestra cada una de las posibilidades al efectuar BD4P4 para el ejemplo de trabajo. Nótese que las permutaciones abordan sólo las cuatro primeras ciudades a visitar, partiendo del origen (ciudad 1). 
Tabla 5. Iteraciones BD4P4 para las primeras cuatro ciudades a visitar.

\begin{tabular}{|c|c|c|c|c|c|c|c|}
\hline \multicolumn{6}{|c|}{ Tours } & $\begin{array}{l}\text { Distancia/ } \\
\text { costo }\end{array}$ & Solución \\
\hline 1 & 5 & 4 & 6 & 3 & 2 & 341 & Vecino más cercano \\
\hline 1 & 3 & 4 & 6 & 5 & 2 & 377 & $\begin{array}{c}1 \text { Intercambio sacrificio } \\
\text { cortoplacista }\end{array}$ \\
\hline 1 & 4 & 3 & 6 & 5 & 2 & 450 & \multirow{23}{*}{$\begin{array}{c}\text { Posibilidades restantes } \\
\text { al efectuar } 4 \mathrm{P} 4 \text { en las } \\
\text { primeras cuatro ciudades } \\
\text { a visitar }\end{array}$} \\
\hline 1 & 4 & 6 & 3 & 5 & 2 & 433 & \\
\hline 1 & 4 & 6 & 5 & 3 & 2 & 458 & \\
\hline 1 & 6 & 4 & 5 & 3 & 2 & 406 & \\
\hline 1 & 6 & b & 4 & 3 & 2 & 423 & \\
\hline 1 & 6 & 5 & 3 & 4 & 2 & 430 & \\
\hline 1 & 5 & 6 & 3 & 4 & 2 & 365 & \\
\hline 1 & 5 & 3 & 6 & 4 & 2 & 348 & \\
\hline 1 & 5 & 3 & 4 & 6 & 2 & 365 & \\
\hline 1 & 3 & 5 & 4 & 6 & 2 & 362 & \\
\hline 1 & 3 & 4 & 5 & 6 & 2 & 379 & \\
\hline 1 & 4 & 3 & 5 & 6 & 2 & 467 & \\
\hline 1 & 4 & 5 & 3 & 6 & 2 & 435 & \\
\hline 1 & 4 & 5 & 6 & 3 & 2 & 443 & \\
\hline 1 & 5 & 4 & 6 & 3 & 2 & 341 & \\
\hline 1 & 5 & 6 & 4 & 3 & 2 & 373 & \\
\hline 1 & 5 & 4 & 3 & 6 & 2 & 350 & \\
\hline 1 & 6 & 4 & 3 & 5 & 2 & 413 & \\
\hline 1 & 6 & 3 & 4 & 5 & 2 & 398 & \\
\hline 1 & 6 & 3 & 5 & 4 & 2 & 398 & \\
\hline 1 & 3 & 6 & 5 & 4 & 2 & 362 & \\
\hline 1 & 3 & 6 & 4 & 5 & 2 & 345 & \\
\hline 1 & 3 & 5 & 6 & 4 & 2 & 377 & \\
\hline
\end{tabular}

Los anteriores cambios en busca de puntos de palanca del sistema se evalúan como en una típica heurística de mejoramiento, actualizando la "mejor" solución encontrada según corresponda. Al efectuar BD4P4 en ningún momento se parte de la mejor solución encontrada al realizar cada permutación, a fin de no limitar la búsqueda desde temprano a localidades. Finalizado este ciclo BD4P4 para el intercambio de "sacrificio cortoplacista", se hace un desplazamiento hacia la derecha para generar más modificaciones al sistema. En la Tabla 6 se ilustra esta situación.

Nótese que 4P4 se desplazó una posición hacia la derecha para generar cambios (propiciar la adaptación evolutiva del sistema) a partir de la segunda ciudad a visitar.

Ha terminado el BD4P4 del primer intercambio "Sacrificio Cortoplacista", encontrándose un apalancamiento del sistema, que permite mejorar la solución del "vecino más cercano" (341). La mejor solución encontrada se actualiza con el tour $(1,3,6,4,2,5,1)$, arrojando una distancia/costo menor (318). Luego se realiza el segundo intercambio "sacrificio cortoplacista", pero esta vez se analiza el impacto de renunciar a la segunda ciudad a
Tabla 6. Iteraciones BD4P4 a partir de la segunda ciudad a visitar, dejando fija la ruta $(1,3)$.

\begin{tabular}{|c|c|c|c|c|c|c|c|}
\hline \multicolumn{6}{|c|}{ Tours } & Distancia/ & Solución \\
\hline 1 & 5 & 4 & 6 & 3 & 2 & 341 & Vecino más cercano \\
\hline 1 & 3 & 4 & 6 & 5 & 2 & 377 & $\begin{array}{c}1 \text { intercambio sacrificio } \\
\text { cortoplacista }\end{array}$ \\
\hline 1 & 3 & 6 & 4 & 5 & 2 & 345 & \multirow{23}{*}{$\begin{array}{c}\text { Posibilidades restantes } \\
\text { al efectuar 4P4 a partir } \\
\text { de la segunda ciudad } \\
\text { a visitar, dejando fija } \\
\text { la ruta }(1,3)\end{array}$} \\
\hline 1 & 3 & 6 & 5 & 4 & 2 & 362 & \\
\hline 1 & 3 & 6 & 5 & 2 & 4 & 420 & \\
\hline 1 & 3 & 5 & 6 & 2 & 4 & 437 & \\
\hline 1 & 3 & 5 & 2 & 6 & 4 & 420 & \\
\hline 1 & 3 & 5 & 2 & 4 & 6 & 383 & \\
\hline 1 & 3 & 2 & 5 & 4 & 6 & 376 & \\
\hline 1 & 3 & 2 & 4 & 5 & 6 & 393 & \\
\hline 1 & 3 & 2 & 4 & 6 & 5 & 343 & \\
\hline 1 & 3 & 4 & 2 & 6 & 5 & 352 & \\
\hline 1 & 3 & 4 & 6 & 2 & 5 & 335 & \\
\hline 1 & 3 & 6 & 4 & 2 & 5 & $318^{*}$ & \\
\hline 1 & 3 & 6 & 2 & 4 & 5 & 320 & \\
\hline 1 & 3 & 6 & 2 & 5 & 4 & 405 & \\
\hline 1 & 3 & 2 & 6 & 5 & 4 & 430 & \\
\hline 1 & 3 & 2 & 5 & 6 & 4 & 428 & \\
\hline 1 & 3 & 2 & 6 & 4 & 5 & 328 & \\
\hline 1 & 3 & 5 & 6 & 4 & 2 & 377 & \\
\hline 1 & 3 & 5 & 4 & 6 & 2 & 362 & \\
\hline 1 & 3 & 5 & 4 & 2 & 6 & 385 & \\
\hline 1 & 3 & 4 & 5 & 2 & 6 & 385 & \\
\hline 1 & 3 & 4 & 5 & 6 & 2 & 379 & \\
\hline 1 & 3 & 4 & 2 & 5 & 6 & 400 & \\
\hline
\end{tabular}

visitar, previamente dada por el vecino más cercano. La Figura 8 ejemplifica este paso.

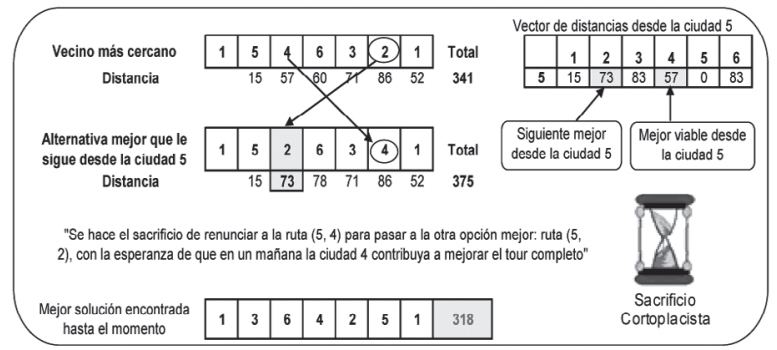

Figura 8. Ejemplo Segundo intercambio "sacrificio cortoplacista".

Fuente: Elaboración propia.

En un momento dado, a medida que el "sacrificio cortoplacista" se hace sobre otras ciudades arrojadas por el "vecino más cercano", puede que la siguiente opción mejor sea una ciudad aún más vecina que la actual, como se ilustra en la Figura 9, donde antes el "vecino más cercano" después de la ciudad 4 tuvo que seleccionarse la ciudad 6 como siguiente viaje, debido a que su ciudad más vecina (5) ya se había asignado. 


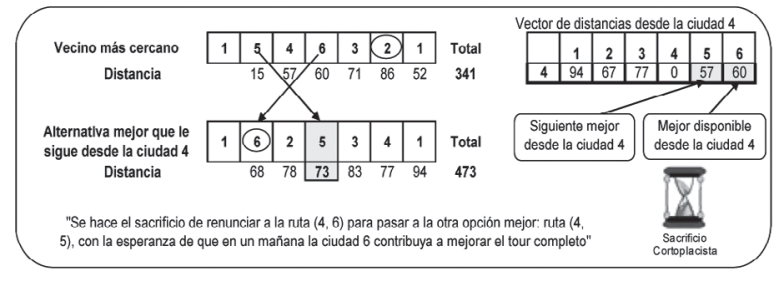

Figura 9. Ejemplo del tercer intercambio de "sacrificio cortoplacista".

El ciclo se repite a través de un simple código hasta que se obtiene una solución, que comprende tan solo un intercambio a nivel de "sacrificio cortoplacista", y lo demás, es una búsqueda local programada con desplazamientos "búsqueda derecha 4P4" para simular el proceso adaptativo del sistema ante el cambio generado.

En la Figura 10 se muestra el seudocódigo del procedimiento general de esta parte del método propuesto.

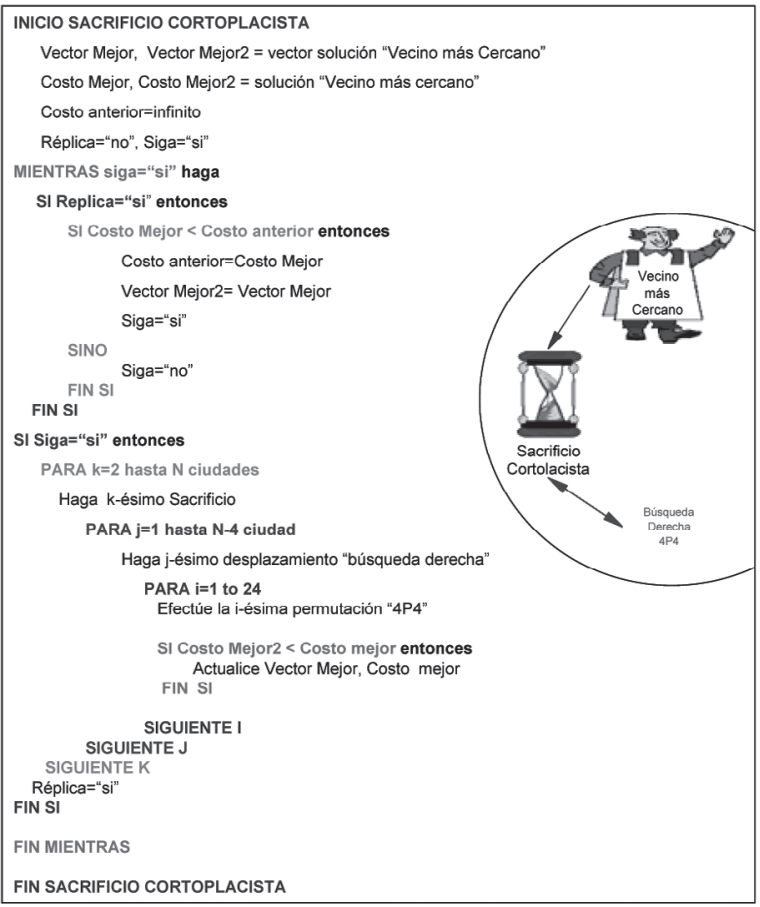

Figura 10. Descripción segunda etapa del método propuesto.

Fuente: Elaboración propia.
En la Figura 10 se nota que cuando la solución del "vecino más cercano" se ha explorado por completo se actualiza la mejor solución encontrada y ésta se somete a prueba efectuando de nuevo el proceso de "sacrificio cortoplacista" hasta que no se encuentre una mejor solución. En este último lapso del procedimiento sí se considera la búsqueda de mejora a nivel de sus vecinos.

\section{- Traslado LIFO}

A partir de la mejor solución arrojada en el paso 2, se procede a explorar posibilidades de mejora mediante "traslado LIFO". Su nombre debido a que las últimas ciudades en recorrer por parte del viajero son las primeras en trasladar, a fin de explorar localidades que mejoren la solución actual (El término LIFO, last in first out, es empleado, comúnmente, como política de inventarios). Luego de cada traslado se efectúa exactamente el mismo procedimiento de apoyo "búsqueda derecha 4P4", igual a como se describió en el paso anterior 2.

En la Figura 11 se ilustran el procedimiento y los traslados efectuados. Estos últimos para una instancia del TSP de 10 ciudades con el ánimo de facilitar la comprensión.

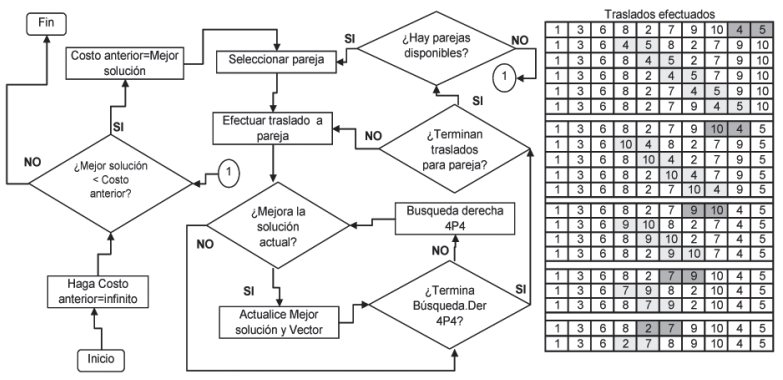

Figura 11. Procedimiento general del paso "Traslado LIFO" y ejemplo de traslados para una instancia TSP de 10 ciudades.

Fuente: Elaboración propia.

En la Figura 11 se nota que el traslado se hace por parejas, comenzando por las ubicadas al fin del recorrido del viajero, que se llevan inicialmente a las posiciones 4 y 5 del vector ciudades y así sucesivamente.

En el procedimiento ofrecido se observa que, al interior del "traslado LIFO", la mejor solución encontrada se va actualizando y, con base en ella, se hacen nuevos traslados; no obstante, cuando se agotan los traslados se abre una pregunta: $i$ el costo/distancia obtenido mediante "traslado LIFO" es menor que la archivada antes de generar este procedimiento?, de ser así el ciclo se repite, si no se detiene. 
Fase 2. Comparación con los procedimientos del WinQSB en distancia/costo y tiempo computacional

Al efectuar análisis de varianza para la distancia/costo arrojada por los diversos métodos sometidos a ensayo se deducen, a partir de la Tabla 7, diferencias significativas entre ellos (valores p por debajo de 0,05 ).

Tabla 7. Análisis de la varianza para distancia/costo.

\begin{tabular}{|lcrccc|}
\hline \multicolumn{1}{|c}{ Fuente } & $\begin{array}{c}\text { Suma de } \\
\text { cuadrados }\end{array}$ & GL & $\begin{array}{c}\text { Cuadrado } \\
\text { Medio }\end{array}$ & Cociente-F & P-Valor \\
\hline EFECTOS PRINCIPALES & & & & & \\
A: Método & $1,09805 \mathrm{E} 6$ & 4 & 274512,0 & 85,38 & 0,0000 \\
B: Ciudades & $1,72713 \mathrm{E} 6$ & 22 & 78505,7 & 24,42 & 0,0000 \\
INTERACCIONES & & & & & \\
AB & 373432,0 & 88 & 4243,55 & 1,32 & 0,0811 \\
RESIDUOS & 369724,0 & 115 & 3214,99 & & \\
\hline TOTAL (CORREGIDO) & $3,56833 \mathrm{E} 6$ & 229 & & & \\
\hline
\end{tabular}

Los cocientes $\mathrm{F}$ están basados en el error cuadrático medio residual.

Llama la atención la presencia de cierta interacción entre los factores método-número de ciudades, con un valor p que excede levemente la significancia del 0.05. En la Figura 12 se muestra la distancia/costo medio arrojado por cada método según los niveles de las ciudades.

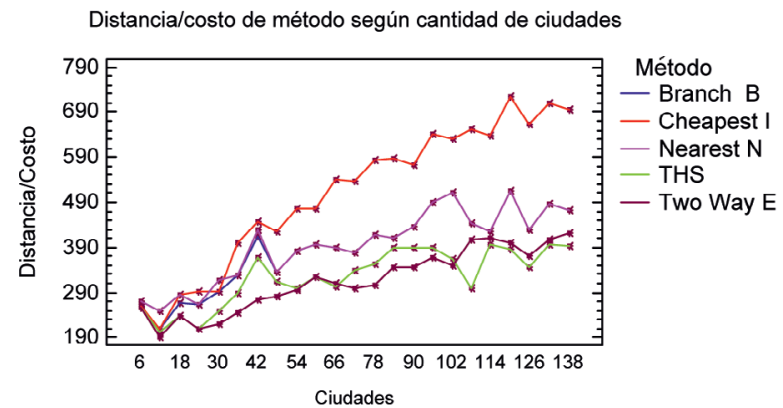

Figura 12. Distancia/costo medio generado por el viajero, según método y tamaño de las instancias del TSP.

Fuente: Elaboración propia.

A partir de la Figura 12 se deduce que en el rango de experimentación (entre 6 y 138 ciudades a incrementos de 6) el método de "cheapest insertion" arroja resultados más desfavorables en términos de costo/distancias que los demás. La heurística de "nearest neighbour" se muestra equivalente al método de "branch and bound" y THS en unos pocos lapsos arroja la misma distancia/costo que el "two-way exchange improvement"; sin embargo, en las primeras instancias (entre 6 y 102 ciudades) este último muestra, comúnmente, su aparente superioridad.

Una sospecha que se genera a partir de la Figura 12 es que el método "two-way exchange improvement" quizá vaya perdiendo consistencia en la eficacia a medida que aumenta la complejidad del problema, de una manera más acelerada que THS, pero es preciso ser cautelosos, ya que esto también podría deberse a la típica aleatoriedad.

Al efectuar rangos múltiples de Duncan, puede concluirse que, desde el punto de vista estadístico y en lo que cobija a la región experimental, las heurísticas "two-way EI" y THS son, en términos de distancia/costo arrojado, más eficaces que los demás, considerándose entre ellos, desde la visión estadística, equivalentes. El segundo lugar, en términos del objetivo de encontrar un tour que en cada caso sea mínimo, le siguen los métodos "branch and bound" y el "nearest neighbour" y finalmente, menos eficaz es "cheapest insertion".

En la Figura 13 se muestra el gráfico de distancia/costo medio arrojado por cada método con intervalos al $95 \%$ de confianza.

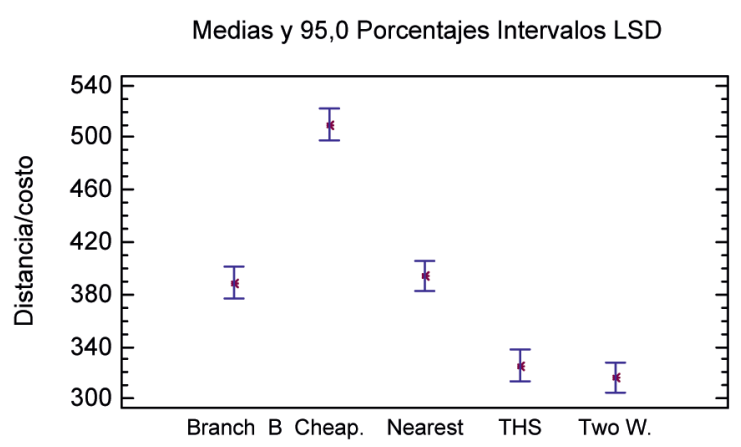

Figura 13. Distancia/costo medio del viajero según método.

Fuente: Elaboración propia.

Si bien desde el punto de vista estadístico THS y su principal "rival" se consideran similares, desde el punto de vista práctico el método "Two-Way EI” puede ser más adecuado, teniendo en cuenta que en un problema real una diferencia mínima en una unidad de longitud puede representar millones en unidades monetarias.

Para corroborar si "two-way exchange improvement" va perdiendo consistencia en la eficacia, de una manera más acelerada que THS, a medida que aumenta la complejidad del problema, se realizaron más experimentos, pero esta vez fijando los niveles de las ciudades desde 120 hasta 142 con incrementos de uno y considerando sólo estos dos métodos.

Como resultado del análisis de varianza, no se encuentran diferencias significativas desde el punto de vista estadístico entre "two-way exchange improvement" y THS, con un valor p muy superior al 0,05 de significancia $(0,4528)$. 
Adoptando la mirada práctica, la situación es contraria (ver Figura 14), pues ahora THS arroja una distancia/costo medio inferior al de "two-way exchange improvement" en 6 unidades aproximadamente, que si representasen en la realidad millones de pesos, aparentemente el decisor podría interesarse, ante mayor complejidad del problema, en emplear este nuevo método.

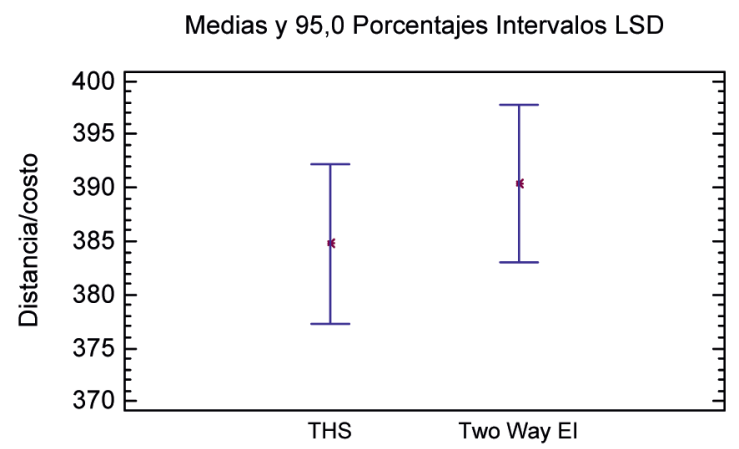

Figura 14. Distancia/costo medio según método (106-142 ciudades).

Fuente: Elaboración propia.

Para analizar un poco más lo que sucede en este nuevo lapso de ciudades, considere la Figura 15. Esto refuerza aún más la sospecha que se tenía: el método "two-way exchange improvement" va perdiendo consistencia en la eficacia a medida que aumenta la complejidad del problema de una manera más acelerada que la THS.

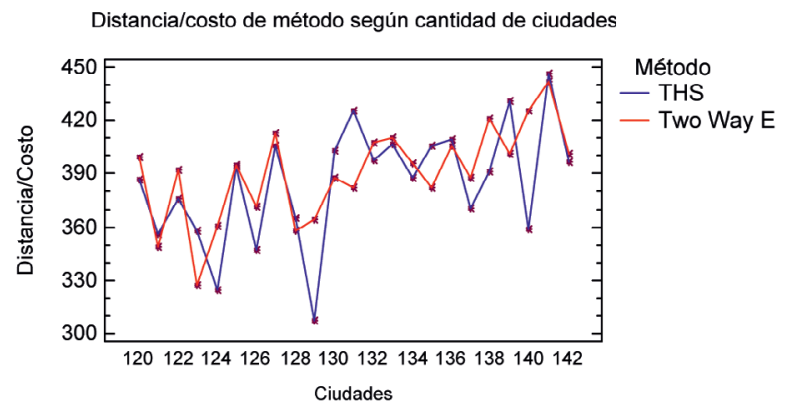

Figura 15. Distancia/costo medio generado por el viajero, según método y tamaño de las instancias (120142 ciudades).

Fuente: Elaboración propia.

Ahora se procederá a observar el comportamiento de la eficiencia de los algoritmos, considerada en función del tiempo computacional empleado para proporcionar la respuesta. Para ello se procedió a analizar el tiempo computacional entre tetraheurística sistémica y el "twoway exchange improvement" también para instancias más complejas del TSP; es decir, entre las mismas 120 y 142 ciudades con incrementos de una, como ocurrió con el análisis de la eficacia.
Los resultados del análisis de varianza para este lapso de complejidad, así como los de Duncan, sorprendieron al equipo investigador, al encontrar diferencias estadísticamente significativas (valor $\mathrm{p}=0,000$ ), mostrando ventajoso, en términos de tiempo de cómputo, a tetraheurística sistémica (Figura 16).

Medias y 95,0 Porcentajes Intervalos LSD. 120-142 ciudades

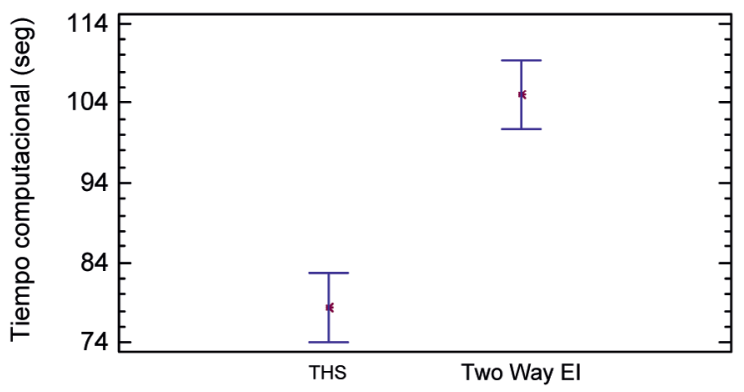

Figura 16. Tiempo medio computacional según método (106-142 ciudades con incremento de a 1). Fuente: Elaboración propia.

El tiempo medio arrojado por THS para las instancias de complejidad abordadas se encuentra en 78,31 segundos, mientras que para "two-way exchange improvement" es de 105,025 segundos. En la Figura 17 se muestran dichos tiempos en función de tamaño de las instancias TSP.

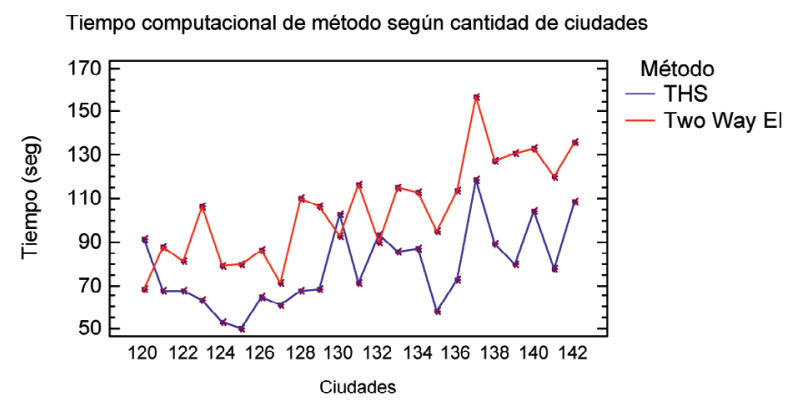

Figura 17. Tiempo medio computacional según método y tamaño de las instancias del TSP (120-142 ciudades con incremento de a 1).

Fuente: Elaboración propia.

Los anteriores análisis reflejan mayor pertinencia de tetraheurística sistémica para resolver instancias del TSP, sobre todo de mayor complejidad algorítmica, destacándose en eficacia y en eficiencia.

\section{Fase 3. Análisis de la proporción de "fracasos" del procedimiento propuesto}

Esta fase está enfocada en estimar y analizar la proporción de fracasos; es decir, cuando "two-way exchange improvement" arroja mejor solución (distancia/costo generado por el viajero) que THS, pues, como se nota en los resultados anteriores, no tiene sentido incluir los demás. 
Igualmente, otra intención con esta fase es analizar el comportamiento de dicha proporción a medida que las instancias del TSP son más complejas (aumentando ciudades). Estimada la proporción de "fracasos", se procedió a graficarla según el tamaño de las ciudades. En la Figura 18 se presenta el comportamiento de dicha proporción.

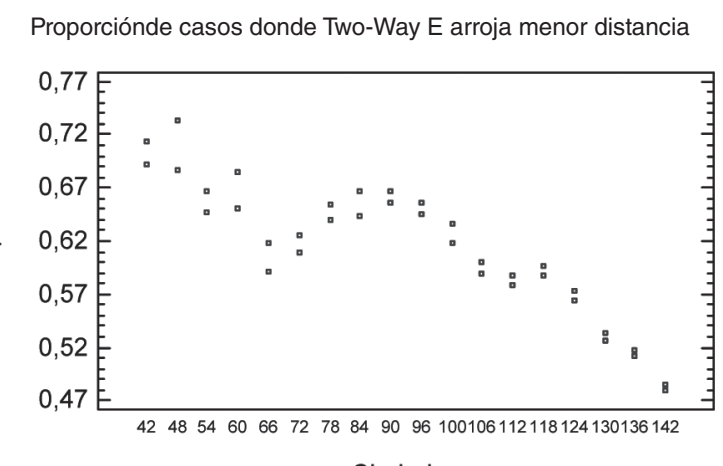

Figura 18. Proporción de casos donde THS “fracasa".

Lo reflejado en la Figura 18 corrobora todas las afirmaciones que se dieron en la fase anterior. Allí puede verse que ante instancias del TSP menos complejas, el método tetraheurística sistémica "fracasa" en mayor proporción, $\mathrm{y}$, a medida que aumenta la complejidad, ésta presenta mayor consistencia en la eficacia que "two-way exchange improvement".

Nótese que ante las instancias del TSP cercanas a 42-54 ciudades la proporción de casos en los que el "two-way exchange improvement" arroja una mejor respuesta que tetraheurística es alrededor de 0,7. Es decir, ante este grado de complejidad del TSP el método desarrollado sólo "vence", en términos de distancia/costo generado por el viajero, en el $30 \%$ de los casos, pero a medida que crece el tamaño de las ciudades la probabilidad de "fracaso" decrece de manera acelerada, hasta el punto de que a partir de 140 ciudades la proporción de éxito comienza a superar el 0,5. En otras palabras, la THS se muestra más consistente en eficacia y en eficiencia que el "two-way exchange improvement" a medida que aumenta la complejidad del TSP.

La ecuación que describe esta relación con un ajuste del $86,5 \%$ (R-cuadrado), considerando 96 datos en el intervalo 30 hasta 142 ciudades, es la siguiente:

$$
p=0,557118+0,0033408 X-0,0000269705 X^{2}
$$

Siendo "p" la proporción de "fracaso" de THS y "X" el número de ciudades de una determinada instancia del TSP.

\section{CONCLUSIONES}

- Con el desarrollo de este proyecto, en el que se genera un trabajo interdisciplinario, se proporcionan elementos que, basados en el pensamiento sistémico, sentido común, creatividad, estadística, arte de la programación y optimización combinatoria, permiten apoyar el proceso de toma de decisiones asociado a los innumerables problemas reales y teóricos a los que tiene aplicación el famoso agente viajero, así como también brinda mecanismos adicionales a los comúnmente empleados que pueden ser utilizados en el ámbito académico para continuar los esfuerzos en buscar respuestas al interrogante, por mucho tiempo existente, ¿cómo obtener la solución óptima al TSP en un tiempo computacional mínimo desde el punto de vista práctico?

- Se hace un análisis, desde el punto de vista sistémico, a la esencia de la heurística "vecino más cercano", la cual es bastante empleada en la cotidianidad de decisiones del ser humano, particularmente en culturas donde prevalece en enfoque cortoplacista. Como resultado se obtiene una estructura sistémica de "Soluciones rápidas que fallan", donde en el corto plazo aparentemente el problema está solucionado, pero a medida que transcurre el tiempo, con presencia de un retardo, se produce un efecto desfavorable que derriba los logros obtenidos con las decisiones rápidas. Este análisis posibilita la creación de una heurística llamada "sacrificio cortoplacista" donde se busca identificar puntos de apalancamiento del sistema en conjunto mediante la renuncia a beneficios rápidos en el corto plazo (distancias inmediatamente cercanas) con la esperanza de que a futuro se tenga un mejor resultado global (una vez finalice el tour).

- La heurística "sacrificio cortoplacista" se enfoca en identificar mediante un "sacrificio" de corto plazo un posible cambio en la solución del "vecino más cercano", de modo que se genere un efecto de apalancamiento que permita disminuir considerablemente y sin mucho esfuerzo la distancia de la solución actual respecto a la óptima del TSP. Es de resaltar que este solo cambio posibilita aprovechar también los beneficios cortoplacistas que produce la visión del "vecino más cercano". Igualmente, aparte del pequeño cambio a producir en el sistema, por medio de la heurística "búsqueda derecha 4P4" se trata de representar los momentos de evolución adaptativa de todo sistema cuando se modifica alguna de sus componentes. Como puede verse, lo anterior tiene suma aplicación a la vida 
cotidiana, haciendo reflexionar una vez más sobre la pertinencia de no descuidar el mediano y largo plazo, pero tampoco ignorar por completo el "hoy". Particularmente en las empresas es precisa la ruptura de paradigmas de pensamiento inclinados sólo en un lado de la balanza, que por ejemplo, cuando se decide disminuir costos, comúnmente se opta por despedir personal, adoptando miopía sobre las consecuencias negativas que se avecinan con retardo.

- Al comparar THS con los cuatro procedimientos incluidos en el WinQSB para solucionar instancias TSP desde el punto de vista de la eficacia, entendiéndose esta como el logro del objetivo: "disminuir la distancia/costo" del tour recorrido por el viajero, al abordar instancias menos complejas del TSP (pocas ciudades), prevalece la primacía del método THS y del "two-way exchange improvement" sobre los demás, siendo estos dos últimos equivalentes desde el punto de vista estadístico. No obstante, cuando la complejidad de las instancias del TSP es mayor (aprox. más de 120 ciudades) se nota una tendencia a la superioridad de THS sobre el "two-way exchange improvement", la cual también es corroborada por el análisis de la proporción de casos en los que el THS es vencido por el "two-way exchange improvement".

- Considerando la eficiencia de THS en comparación con los demás, entendiéndose ésta como la racionalización del recurso "tiempo computacional", las heurísticas "nearest neighbour" y "cheapest insertion" se destacan, ocupando el último lugar, entre los métodos sometidos a ensayo, THS y "two-way exchange improvement", los cuales también para instancias menos complejas del TSP (menor número de ciudades) se consideran con tiempo computacional equivalente. Sin embargo, a pesar de que, como era de esperarse, estos dos últimos métodos arrojan mayores tiempos computacionales que los demás, son más eficaces en las respuestas y bastante viables en tiempo, desde el punto de vista práctico.

- Se resalta que, cuando la complejidad de las instancias del TSP incrementa, es decir, se tienen más ciudades (específicamente este análisis consideró instancias entre 120-142 ciudades con incrementos de a una) el THS desde el punto de vista práctico se muestra superior en eficacia al mejor ("two-way exchange improvement”) de los demás sometidos a prueba, y desde el punto de vista estadístico y práctico desde luego, el THS es también muy superior en términos de eficiencia computacional. En otras palabras, a medida que aumenta la complejidad de los problemas el THS presenta mayor consistencia en la eficacia y en la eficiencia que "two-way exchange improvement".

\section{PROYECCIONES}

- A modo de exploración, mediante el ajuste de una relación entre la proporción de casos en los que el método "nuevo" fracasa, con un ajuste del $86,5 \%$, considerando normales los errores estándar de las componentes del modelo, se simuló para instancias de hasta 200 ciudades, arrojando supremacía del método "nuevo" sobre el "two-way exchange improvement". Esta situación motiva al equipo investigador a experimentar con una región de instancias mucho más complejas del TSP no sólo comparándose con el "two-way exchange improvement", sino también con otros métodos, a fin de corroborar la consistencia en eficacia y en eficiencia fuera de la región experimental, a medida que la situación se asemeja a un problema real (crece su complejidad).

- $\quad$ Surge el interés de estudiar posibles estructuras sistémicas de heurísticas existentes, de modo que, comprendiendo más a fondo el sistema, sea más viable encontrar una solución que mejore el beneficio en variables de interés y en un momento dado y disminuya las consecuencias negativas en otras variables o lapsos temporales.

- Es importante estudiar cómo pueden aplicarse los diversos elementos que aporta este trabajo a otros problemas de la optimización combinatoria. Así como analizar, bajo una mirada sistémica, sus aplicaciones y enseñanzas a las demás decisiones a las que se enfrenta el ser humano en la vida cotidiana.

El proyecto con el que continuará el equipo investigador será el de comparar el método desarrollado con resultados arrojados por las metaheurísticas Colonia de Hormigas y Algoritmos Genéticos. Igualmente, se analizará el efecto de incluir en el método desarrollado una componente aleatoria, típica en metaheurísticas.

\section{REFERENCIAS}

[1] J. Arreola y A. Arreola. "Programación lineal: Una introducción a la toma de decisiones cuantitativa". Thomson Ed. México. 2002.

[2] R. Quirós. “Optimización del proceso de aserrío en madera de cortas dimensiones en el Pacífico Seco, Costa Rica". Tesis para optar al grado de Magíster. Turrialba, Costa Rica. 1990. 
[3] E. Vallada, C. Maroto, R. Ruiz y B. Segura. "Análisis de la programación de la producción en el sector cerámico español". Boletín de la Sociedad Española de Cerámica y Vidrio. Vol. 44 No 1, pp. 39-44. 2005.

[4] J. Pérez, C. Parra y D. Torres. "Modelación y simulación computacional de un proceso productivo de una pequeña empresa usando dinámica de sistemas". Ingeniería y Desarrollo. No 20, pp. 151171. 2006.

[5] R. Álvarez, A. Corberán y J. Tamarit. "La combinatoria poliédrica y el problema del viajante. Aplicación al caso de ciento tres ciudades Españolas”. Qüestió. Vol. 9 № 3, pp. 199-213. Septiembre 1985.

[6] R. Marti. "Procedimientos metaheurísticos en optimización combinatoria". Departament d'Estadística i Investigació Operativa, Facultat de Matemàtiques, Universitat de Valèencia, p. 4. 2005.

[7] V. Quesada y J. Vergara. "Análisis Cuantitativo con WINQSB”. Ed. Juan Carlos Martínez Coll. Administración Industrial. Universidad de Cartagena. 2006.

[8] S. Herbert and A. Newell. "Heuristic Problem Solving: The Next Advance in Operations Research". Operations Research. Vol. 6, Issue 1, pp. 1-10. January-February, 1958.

[9] D. Arana, A. Medaglia y F. Palacios. "Herramienta para el diseño óptimo de la red de rutas en sistemas de transporte masivo con aplicación a Transmilenio S.A.” Memos de Investigación. IIND 2004-026.

[10] P. Priore, J. Puente, D. García y A. Gómez. "Planificación de turnos de trabajo en el servicio de urgencias de un hospital mediante algoritmos genéticos". Revista europea de dirección y economía de la empresa. Vol. 10 No 2, pp. 57-66. 2001.

[11] D. Holstein. "Una Metaheurística Coevolutiva para el problema del viajante de Comercio". Tesis de Grado. Universidad Nacional de la Plata, p. 5. 1998.

[12] R. Pastor y A. Corominas. "Estrategias de resolución del problema de flow-shop tipo p, en el marco de los procedimientos branch and bound". Boletín de la SEIO. Vol. 16 No 30, p. 59. 2004.

[13] J. González y R. Ríos. "Investigación de operaciones en acción: Aplicación del TSP en problemas de manufactura y logística". Ingenierías. Vol. $2 \mathrm{~N}^{\circ} 4$, pp. 19-20. 1999.

[14] M. Flood. "The Travelling Salesman Problem". Operations Research. Vol. 4, Issue 1, pp. 61-75. 1956.
[15] R. Marti. "Procedimientos metaheurísticos en optimización combinatoria". Departament d'Estadística i Investigació Operativa, Facultat de Matemàtiques, Universitat de Valèencia, p. 12. 2003.

[16] A. Pavez y H. Acevedo. "Un algoritmo ACS con selección dinámica de movimiento y operador 2-opt”. Ingeniería informática. No 8. 2002.

[17] G. Croes. "A Method for Solving Traveling-Salesman Problems". INFORMS Operations Research. Vol. 6, Issue 6, pp. 791-812. November-December, 1958.

[18] D.E. Rosenkrantz, R.E. Stearns and P.M. Lewis. "An Analysis of Several Heuristics for the Traveling Salesman Problem". SIAM Journal on Computing. Vol. 6, pp. 563-581. 1977.

[19] I. Oliver, D. Smith y J. Holland. "A study of permutation crossover operators on the traveling salesman problem". Proceedings of the Second International Conference on Genetic Algorithms on Genetic algorithms and their application. Cambridge, Massachusetts, United States, pp. 224230. 1987.

[20] J. González y R. Ríos. "Investigación de operaciones en acción: Aplicación del TSP en problemas de manufactura y logística". Ingenierías. Vol. 2 No 4 , pp. 21-22. 1999.

[21] R. Marti. "Procedimientos metaheurísticos en optimización combinatoria". Departament d'Estadística i Investigació Operativa, Facultat de Matemàtiques, Universitat de Valèencia, pp. 11-12. 2003.

[22] F. Etchegaray. "Optimización del proceso de teñido de telas". Universidad Nacional de Quilmes, Tesis PhD., p. 44. Argentina. 2005.

[23] E. Silver, R. Victor, V. Vidal and D. de Werra. "A tutorial on heuristic methods". European Journal of Operational Research. Vol. 5, Issue 3, pp. 153162. 1980.

[24] R. Marti. "Procedimientos metaheurísticos en optimización combinatoria". Departament d'Estadística i Investigació Operativa, Facultat de Matemàtiques, Universitat de Valèencia, p. 6. 2003.

[25] F. Maldonado, R. Radillo, G. Baldoquin and, A. Ruiz. "Optimization of the Colour Sequence in the Dyeing Process: washing the equipment". Coloration Technology. Vol. 121, Issue 3, pp. 164168. May, 2005.

[26] D. Rosenkrantz, R. Stearns and P. Lewis. "An analysis of several heuristics for the traveling salesman problem". SIAM Journal on Computing. Vol. 6, Issue 3, pp. 563-581. 1977. 
[27] L. Bodin, B. Golden, A. Assad and M. Ball. "Routing and scheduling of vehicles and crews: the state of the art". Computers \& Operations Research. Vol. 10, pp. 63-212. 1983.

[28] S. Chanta and S. Ornurai. "Application of AHP for Travelling Salesman Problem". Proceedings of the International Conference on Computer and Industrial Management. ICIM, pp. 16. 1-16.5. Bangkok, Thailand. October 29-30, 2005.

[29] S. Lin and B. Kernighan. "An effective heuristic for the travelling salesman problem". Operations Research. Vol. 21, pp. 498-516. 1973.

[30] R. Martí. "Algoritmos Heurísticos en Optimización Combinatoria". Departament d'Estadística i Investigació Operativa, Facultat de Matemàtiques, Universitat de Valèencia, pp. 17-19. Valencia, España. 2005.

[31] J. Lenstra and A. Rinooy. "Some simple applications of the travelling salesman problem". Operational Research Quarterly Press. Vol. 26, Issue 4, Part 1, pp. 717-718. November, 1975.

[32] J. Potvin. "The Travelling Salesman Problem: A Neural Network Perspective". ORSA Journal of Computing. Vol. 5, pp. 328-348. 1993.
[33] H. Taha. "Investigación de Operaciones". Pearson Prentice Hall, $7^{\text {th }}$ Edition, p. 390. México. 2004.

[34] H. Taha. "Investigación de Operaciones". Pearson Prentice Hall, $7^{\text {th }}$ Edition, pp. 394-395. México. 2004.

[35] R. Martí. “Algoritmos Heurísticos en Optimización Combinatoria”. Departament d'Estadística i Investigació Operativa, Facultat de Matemàtiques, Universitat de Valèencia, p. 9. Valencia, España. 2005.

[36] M. Howell. "Actionable performance measurement: a key to success". American Society for Qualit. Edición Ilustrada, p. 4. 2006.

[37] D. Montgomery. "Diseño y análisis de experimentos". Limusa Wiley, 2 ${ }^{\text {da }}$ Edición, pp. 174-177. México. 2004.

[38] D. Montgomery. "Diseño y análisis de experimentos". Limusa Wiley, 2da Edición, pp. 60-75; 177-184. México. 2004.

[39] D. Duncan. "Multiple range and multiple F tests". Biometrics. Journal of the International Biometric Society. Vol. 11, pp. 1-42. 1955. 